\title{
Prognostic factors in the management of osteosarcoma
}

\author{
Oboirien M*, Agbo SP, Ajiboye LO, Galadima BA and Nwashili RC \\ Department of Surgery, Usmanu Danfodiyo University Teaching Hospital, Sokoto, Nigeria
}

\begin{abstract}
Background: Osteosarcoma remains a challenging condition to treat especially in resource poor countries. With recent advances in limb salvage procedures and chemotherapy, hopes for improved survival in patients have been renewed. Problems of late presentation, delay diagnosis, lack of resources for treatment affects the outcome of patients in our sub-region.

Objectives: We aim to document the prognostic factors determining outcome in patients with osteosarcoma in our sub-region.

Methodology: It was a retrospective study of cases of osteosarcoma seen at a tertiary hospital in North west Nigeria from January 2014 and June 2015 . A pro-format was used to obtain data from patients' clinical records, ward records, and theatre operation register. Statistical analysis was done with IBM SPSS 20.

Results: There were twenty-one cases of histological confirmed osteosarcoma with age range of between 7 and 35 years and a mean age of $20.2 \pm 7.90$. There were 15 (71.4\%) males and 6 (28.6\%). All the patients were of the Hausa/Fulani ethnic group. Seventy-one percent of patient were of low socioeconomic status. Majority of patients (90.5\%) presented with pain and swelling of the affected limb and $71.4 \%$ presented within 6 months of onset. The distal femur was the most affected in $52.4 \%$ of cases. Cross tabulation and correlation of sex, socioeconomic status, duration of symptoms, region affected and Enneking stage of the disease with the disease showed a statistical significance of $\mathrm{p}=0.005$ (sex) and $\mathrm{p}=0.03$ (Enneking stage). There was a mortality rate of $19 \%$ with fate of $48 \%$ of patients were unknown.

Conclusion: The study showed that most patients with osteosarcoma were young male individuals of low socioeconomic status with advance disease and a poor prognostic outlook.
\end{abstract}

\section{Introduction}

Osteosarcoma is a malignant osteoid producing tumour and is the commonest primary bone tumour affecting mainly children and adolescents in the second decade of life [1]. There are many variants with contrasting features and prognosis. The classic variety which is highly malignant constitute more than $75 \%$ of cases and is common in younger age group (8-25 years) while the low grade parosteal variant is common in older age group (10-45 years) [1,2]. Secondary osteosarcomas occur in bones affected by preexisting abnormalities such as Paget's disease, radiation lesions, bone infarct, fibrous dysplasia or osteomyelitis [3]. Among primary osteosarcomas, these precursor conditions are not observed. Among older children and adolescents (12-18 years), they are the third most common type of neoplasia, preceded by leukaemia and lymphoma. Their etiology remains unknown, but it is believed that the period of their greatest incidence coincides with the growth spurt of puberty. This period is characterized by rapid growth of the long bones, suggesting a correlation between this phase $[3,4]$.

It remains a challenging condition to treat especially in resource poor countries. With recent advances in limb salvage procedures and chemotherapy, hopes for improved survival in patients have been renewed. Problems of late presentation, delay diagnosis, lack of resources for treatment and follow up affects the outcome of patients in our sub-region. We aim to document the prognostic factors determining outcome in patients with osteosarcoma in our sub-region.

\section{Methodology}

It was a retrospective study of cases of osteosarcoma seen at a tertiary hospital in North west Nigeria from January 2014 and June 2015. A pro-format was used to obtain data from patients' clinical records, ward records, and theatre operation register. These were patients seen and managed in our facilities. Statistical analysis was done with IBM SPSS 20 and results presented in tabular and graphical forms.

\section{Results}

There were twenty-one cases of histological confirmed osteosarcoma with age range of between 7 and 35 years and a mean age of $20.2 \pm$ 7.90. There were $15(71.4 \%)$ males and $6(28.6 \%)$ with a M:F ratio of 2.5:1 (Figure 1). All the patients were of the Hausa/Fulani ethnic group. Seventy-one percent of patient were of low socioeconomic status. Most

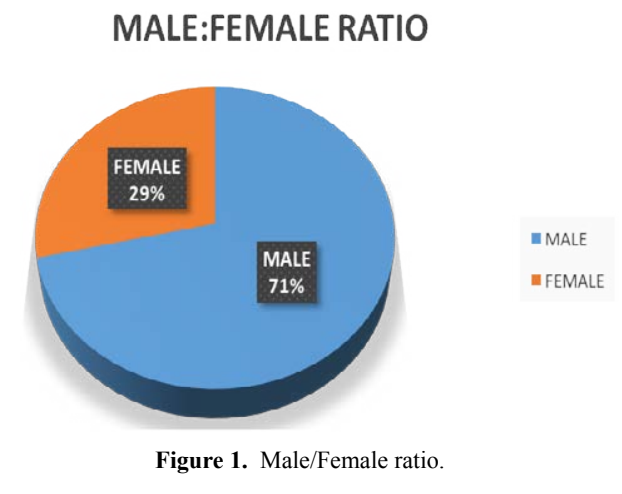

Correspondence to: Oboirien Muhammad, Department of Surgery, Usmanu Danfodiyo University Teaching Hospital, Sokoto, Nigeria, E-mail: moboirien@yahoo.com

Key words: osteosarcoma, prognostic factors, outcome

Received: April 25, 2017; Accepted: May 12, 2017; Published: May 15, 2017 
of the patients (90.5\%) presented with pain and swelling of the affected limb while pathological fractures and bleeding ulcerations were seen in $4.8 \%$ cases respectively (Figure 2 ). The duration of symptoms was such that $71.4 \%$ presented within 6 months of onset while $28.6 \%$ presented between 6 to 12 months after the onset of symptoms. The distal femur was the most affected in $52.4 \%$ of cases followed by the proximal tibia $19 \%$, proximal humerus $19 \%$, pelvis $4.8 \%$ and distal tibia $4.8 \%$. Radiological evaluation showed that $90.5 \%$ were Osteoblastic and 9.5\% were Osteolytic. Enneking staging showed that $81 \%$ were stage 3 and $19 \%$ were stage 2 . Thirty-three per cent of patient had a form of amputation while $67 \%$ had no surgery or limb salvage. Twenty-four per cent of the patients had adjuvant chemotherapy while $9.3 \%$ had neo-adjuvant chemotherapy (Table 1). Follow-up was done for up to 12 months during which $67 \%$ were loss to follow-up and only $19 \%$ were regular on follow-up. The mortality showed that 4 (19\%) were dead at 3 months and the fate of 10 (48\%) were unknown. Thirty-three per cent were alive at six months of follow-up. Cross tabulation and correlation of sex, socioeconomic status, duration of symptoms, region affected and Enneking stage with the disease showed a statistical significance of $\mathrm{p}=0.005$ (sex) and $\mathrm{p}=0.03$ (Enneking stage) (Table 2).

\section{Discussion}

Clinical studies that aims to identify prognostic factors in the overall survival of patients with osteosarcoma have been published but results have been varied as a result of non harmonisation of the various methodologies [5]. The number of cases seen in our study over a 16 month period showed an average of 1.3 cases per month. This is an indication of the rarity of the condition as our facility being a referral centre with a radiotherapy facility is sufficiently equipped to manage these cases in the sub-region. Overall, the age distribution of these tumours in our study was unimodal unlike the bimodal pattern described in the literature $[3,6]$. The first and larger peak of incidence is found to occur during the second decade of life and the average age of the patients in our series was in the second decade. Patients with primary osteosarcoma are in the first peak and the results from our series are concordant with this picture. The second and smaller peak is seen among patients over 40 years of age, and has been found to be secondary to a pre-existing bone abnormality like pagets' disease. The variables of age and gender have been shown to be prognostic factors

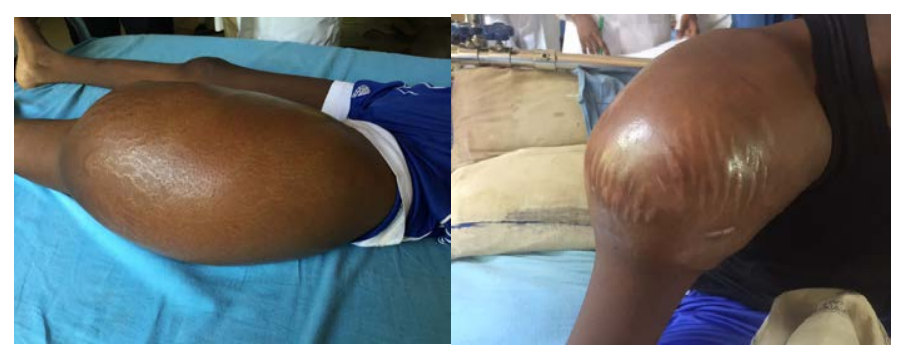

Figure 2. Presentation.

Table 1. Showing the treatment modalities offered.

\begin{tabular}{|l|c|c|}
\hline Treatment & Frequency & Per cent \\
\hline Chemotherapy & 2 & 9.5 \\
\hline Neoadjuvant & 5 & 23.8 \\
\hline Adjuvant & 14 & 66.7 \\
\hline None & \multicolumn{2}{|}{} \\
\hline Surgery & 14 & 67 \\
\hline Amputation & 7 & 33 \\
\hline No Surgery & & \\
\hline
\end{tabular}

Table 2. Correlation between sex, socioeconomic status, duration of symptoms, region affected, Enneking stage with mortality.

\begin{tabular}{|c|c|c|c|c|c|}
\hline & \multicolumn{3}{|c|}{ Mortality } & \multirow{2}{*}{ Total } & \multirow{2}{*}{$\begin{array}{c}\text { Chi } \\
\text { Square }\end{array}$} \\
\hline & $<3$ months & Unknown & $\begin{array}{c}\text { Alive at } 6 \\
\text { months }\end{array}$ & & \\
\hline \multicolumn{6}{|l|}{ Sex } \\
\hline Male & 4 & 10 & 1 & 15 & \multirow[t]{2}{*}{$0.005 \mathrm{df}=1$} \\
\hline Female & 0 & 0 & 6 & 6 & \\
\hline \multicolumn{6}{|c|}{ Socioeconomic Status } \\
\hline Low SES & 3 & 6 & 6 & 15 & \multirow{2}{*}{$\begin{array}{l}0.75 \\
d f=1\end{array}$} \\
\hline Middle SES & 1 & 4 & 1 & 6 & \\
\hline \multicolumn{6}{|c|}{ Duration of Symptoms } \\
\hline$<6$ Months & 3 & 5 & 7 & 15 & \multirow{2}{*}{$\begin{array}{l}0.44 \\
d f=1\end{array}$} \\
\hline 6-12 Months & 1 & 5 & 0 & 6 & \\
\hline \multicolumn{6}{|l|}{ Region Affected } \\
\hline Proximal humerus & 0 & 3 & 1 & 4 & \multirow{5}{*}{$\begin{array}{l}0.43 \\
\mathrm{df}=1\end{array}$} \\
\hline Distal femur & 3 & 5 & 3 & 11 & \\
\hline Proximal tibia & 0 & 1 & 3 & 4 & \\
\hline Distal tibia & 0 & 1 & 0 & 1 & \\
\hline Pelvis & 1 & 0 & 0 & 1 & \\
\hline \multicolumn{6}{|l|}{ Enneking stage } \\
\hline Stage 2 & 0 & 0 & 4 & 4 & \multirow{2}{*}{$\begin{array}{l}0.03 \\
d f=1\end{array}$} \\
\hline Stage 3 & 4 & 10 & 3 & 17 & \\
\hline
\end{tabular}

in some series using univariate analysis alone [5]. However, some studies have stated that male gender was a poor prognostic factor and others have indicated that age significantly influenced the survival of their patients in a double analysis (univariate and bivariate) [6]. In the present study, age was not found to be a significant prognostic variable, in agreement with other studies but sex was found to be significant $(\mathrm{p}=0.005)$ with the male gender having a poorer prognosis.

All the patients in our series were of a particular ethnic group and this may be reason for further research as other ethnic groups also resides in this region. Perception about the disease coupled with other social factors may determine overall survival in patients with the disease. Socioeconomic status (SES) being a measure of education, income and occupation has been shown to be a strong predicator of an individuals' morbidity or mortality with regards to diseases with education being the most significant factor [7]. Individuals and caregiver with low SES have difficulty in understanding the disease entity. This is also responsible for the delay in presentation, patronage of traditional bone setters (TBS) and refusal to accept treatment options. Patronage of TBS for all forms of musculoskeletal diseases abound in this region thereby delaying proper diagnosis and treatment [8].

The duration of presentation did not have a significant correlation with mortality and this has been collaborated by other studies which suggest that the stage of the disease at the time of its initial presentation depends more on the biological properties of the tumor than on late diagnosis. It is however important to state that early presentation, diagnosis and institution of treatment in tumours that has increase tendencies for metastasis will improve metastasis free survival $[6,9]$.

Review of the literature shows that the femur is the most frequent site, followed by the tibia and humerus. In the our study, we also found that these sites were the most affected locations. Some studies have demonstrated that axial tumours as a prognostic factor have a relatively greater adverse effect than do those of the appendicular skeleton as the only patient with pelvic tumour in our series died during evaluation. Axial tumours may cause a diagnostic dilemma as result of their location [10-12]. With regards to the long bone, some authors reported that the prognosis for tumours located in the femur was worse $[13,14]$. 
By contrast, some authors observed a worse prognosis for tumours in the humerus [15]. Our study did not show any significant relationship between the anatomical site of tumour and mortality $(\mathrm{p}=0.43)$.

Clinical complaints of pain and swelling were frequently encountered. Pain is considered to be a warning symptom, except in cases of pathological fracture $[10,11]$. In our study, pain was present in all the patients with $5 \%$ each having a pathological fracture or bleeding ulcer. Our pathological fracture rate of $4.8 \%$ was less than the rate of 7 to $17 \%$, in the literature. The Enneking stage of the tumour had a significant relationship with mortality $(\mathrm{p}=0.03)$. Stage 3 Enneking denotes a metastatic disease and the aim of treatment was palliative [1619]. Local regional control was achieved through radical surgery with little chance for limb salvage. Chemotherapy rate was less than $50 \%$ either from outright refusal by patients or that they were too poor to afford the chemotherapeutic agent. The patients that had neo-adjuvant and adjuvant chemotherapy did well during the 12 months followup. The presence of metastases is directly related to a worse prognosis regarding survival [20-23]. A mortality rate of $19 \%$ was confirm in our study even though a significant number of patients' were lost to follow up.

A major limitation to our study was the high number of patients that were lost to follow-up. The major reason being that most do not have the resources to undertake treatment in a society with no health insurance while others may have succumbed to the disease

\section{Conclusion}

The study showed that most patients with osteosarcoma are young males individuals, low socioeconomic class with advance disease and a poor prognostic outlook. Health education on the need to seek help for all forms of musculoskeletal disease would ensure that osteosarcoma is diagnosed early and treatment instituted. Chemotherapeutic agents needs to be subsidize by the government.

\section{References}

1. Provisor AJ, Ettinger LJ, Nachman JB, Krailo MD, Makley JT, et al. (1997) Treatment of nonmetastatic osteosarcoma of the extremity with preoperative and postoperative chemotherapy: a report from Children's Cancer Group. J Clin Oncol 15: 76-84. [Crossref]

2. Link MP, Gebhardt MC, Eilber F (2002) Osteosarcoma. In: Pizzo, PA; Poplack, DG. Principles and Practice of Pediatric Oncology. Philadelphia: Lippincott Williams \& Wilkins; 4th Edition pp. 889-920.

3. Dahlin DC, Coventry MB (1967) Osteogenic sarcoma. A study of six hundred cases. $J$ Bone Joint Surg Am 49: 101-110. [Crossref]

4. Petrilli AS, Gentil FC, Epelman S, Lopes LF, Bianchi A, et al. (1991) Increased survival, limb preservation, and prognostic factors for osteosarcoma. Cancer 68: 733737. [Crossref]

5. Júnior RZB, de Camargo OP (2009) Prognostic Factors in the Survival of Patients Diagnosed with Primary Non-Metastatic Osteosarcoma with a Poor Response to Neoadjuvant Chemotherapy. Clinics 64 (12): 1177-1186.

6. Ferrari S, Bertoni F, Mercuri M, Picci P, Giacomini S, et al. (2001) Predictive factors of disease- free survival for nonmetastatic osteosarcoma of the extremity: an analysis of 300 patients treated at the Rizzoli Institute. Ann Oncol 12:1145-1150. [Crossref]

7. Winkleby MA, Jatulis DE, Frank E, Fortmann SP (1992) Socioeconomic status and health: how education, income, and occupation contribute to risk factors for cardiovascular disease. Am J Public Health 82(6): 816-820. [Crossref]

8. Oboirien M, Khalid A (2013) Knowledge and Belief about Traditional Bone Setters' practices in Sokoto, North-West Nigeria. The Internet journal of Orthopaedic Surgery 21(2).

9. Petrilli AS, de Camargo B, Filho VO, Bruniera P, Brunetto AL, et al. (2006) Results of the Brazilian Osteosarcoma treatment group studies III and IV: prognostic factors and impact on survival. J Clin Oncol 24: 1161-1168.

10. Rech A, Castro CG Jr, Mattei J, Gregianin L, Di Leone L, et al. (2004) [Clinical features in osteosarcoma and prognostic implications]. J Pediatr (Rio J) 80: 65-70. [Crossref]

11. Simon R (1978) Clinical prognostic factors in osteosarcoma. Cancer Treat Rep 62: 193-197. [Crossref]

12. Bacci G, Ferrari S, Bertoni F, Ruggieri P, Picci P, et al. (2000) Long-term outcome for patients with nonmetastatic osteosarcoma of the extremity treated at Istituto Ortopedico Rizzoli according to the Instituto Ortopedico Rizzoli/osteosarcoma-2 protocol: an updated report. J Clin Oncol 18: 4016-4027. [Crossref]

13. Glasser DB, Lane JM, Huvos AG, Marcove RC, Rosen G (1992) Survival, prognosis and therapeutic response in osteogenic sarcoma. The Memorial Hospital experience. Cancer 69: 698-708. [Crossref]

14. Pakos EE, Nearchou AD, Grimer RJ, Koumoullis HD, Abudu A, et al. (2009) Prognostic factors and outcomes for osteosarcoma: an international collaboration. Eur J Cancer 45: 2367-2375. [Crossref]

15. Goorin AM, Perez-Atayde A, Gebhardt M, Andersen JW, Wilkinson RH, et al. (1987) Weekly high-dose methotrexate and doxorubicin for osteosarcoma: The Dana-Farber Cancer Institute/The Children 's Hospital-Study III. J Clin Oncol 5: 1178-1184. [Crossref]

16. Enneking WF, Spanier SS, Goodman MA (1980) Current concepts review. The surgical staging of musculoskeletal sarcoma. J Bone Joint Surg Am 62: 1027-1030. [Crossref]

17. Davis AM, Bell RS, Goodwin PJ (1994) Prognostic factors in osteosarcoma: a critical review. J Clin Oncol 12: 423-431. [Crossref]

18. Saeter G, Elomaa I, Wahlqvist Y, Alvegård TA, Wiebe T, et al. (1997) Prognostic factors in bone sarcomas. Acta Orthop Scand Suppl 273: 156-160. [Crossref]

19. Hauben EI, Weeden S, Pringle J, Van Marck EA, Hogendoorn PC (2002) Does the histological subtype of high-grade central osteosarcoma influence the response to treatment with chemotherapy and does it affect overall survival? A study on 570 patients of two consecutive trials of the European Osteosarcoma Intergroup. Eur J Cancer 38: 1218-1225. [Crossref]

20. Raymond AK, Chawla SP, Carrasco CH, Ayala AG, Fanning CV, et al. (1987) Osteosarcoma chemotherapy effect: A prognostic factor. Semin Diagn Pathol 4: 212236. [Crossref]

21. Spanier SS, Shuster JJ, Vander Griend RA (1990) The effect of local extent of the tumor on prognosis in osteosarcoma. J Bone Joint Surg Am 72: 643-653. [Crossref]

22. Bieling P, Rehan N, Winkler P, Helmke K, Maas R, et al. (1996) Tumor size and prognosis in aggressively treated osteosarcoma. J Clin Oncol 14: 848-858. [Crossref]

23. Bacci G, Ferrari S, Longhi A, Forni C, Zavatta M, et al. (2002) High grade osteosarcoma of the extremity: Differences between localized and metastatic tumors at presentation. $J$ Pediatr Hematol Oncol 24: 27-30. [Crossref]

Copyright: (C2017 Oboirien M. This is an open-access article distributed under the terms of the Creative Commons Attribution License, which permits unrestricted use, distribution, and reproduction in any medium, provided the original author and source are credited. 